\title{
Position of the Sapheno-femoral Junction; to what Extent is Ideal "In Situ" Saphenous Vein Graft Possible?
}

\author{
By \\ Masahiro TOSHIMA*, Harumichi SHINOHARA $\dagger^{* *}$ and Shigeki MIZUKAMI***
*Department of Surgery, Toyama Kenritsu-Chyuou Hospital
**Department of Anatomy I, Faculty of Medicine, Toyama Medical and Pharmaceutical University, Sugitani 2630, Toyama 930-01
***Department of Anatomy, Fukui Prefectural College \\ †To whom correspondence should be addressed \\ - Received for Publication, February 27, 1990-

\begin{abstract}
Key words: Sapheno-femoral Junction, "In Situ" saphenous graft
Summary: The relative position of the sapheno-femoral junction (SFJ) with respect to the origin of the arterial trunk from which the deep femoral artery arises was examined. Among 47 SFJ's, $9(19 \%)$ were located at a proximal level to the arterial trunk, another $9(19 \%)$ were located at an equal level to the trunk, and the remaining $29(62 \%)$ were located at a distal level to the trunk. "In situ" saphenous vein graft for occlusion of the femoral artery is best indicated when the SFJ is
\end{abstract} \\ located proximal to the arterial trunk. Our results suggested that such cases are encountered in as few as $19 \%$ of patients.
}

During a previous study on the distribution of valves in the great saphenous vein, we noticed that the relative position of the sapheno-femoral junction with respect to the origin of the dee femoral artery varied considerably among individuals and even between the right and left sides of a single individual. This finding was a surprise and appeared to warrant further investigation, since the relative position is one of the most important factors determining whether a patient with femoral artery occlusion is indicated to have "in situ" great saphenous vein graft or not (see DISCUSSION for details). Unfortunately, there is no previous anatomical contribution on this topic. We therefore undertook an examination of 47 sapheno-femoral junctions in 25 cadavers.

\section{Materials and Methods}

Forty-seven sapheno-femoral junctions (SFJs) of 25 cadavers were investigated.

The patterns of branching from the proximal part of the femoral artery have been well documented by some anatomists (Adachi, 1928; Emura et al., 1985; Emura et al., 1989). These authors categorized the patterns into eight, including some subdivisions. "In situ" graft operation is usually performed regarding the deep femoral artery as a landmark to determine the level of arteriovenous anastomosis. For this reason, we classified the branching patterns into three types on a practical basis. In type 1 , the deep femoral artery branches off from the femoral artery with the lateral and medial circumflex arteries forming a common trunk. In type 2, the deep femoral artery branches off from the femoral artery with either one of the lateral or the medial circumflex artery for their branches) forming a common trunk. In type 3 , the deep femoral artery branches off separately from other arteries.

In our study, all branching patterns were found to belong to type 1 or type 2 (see RESULTS), so that the distance between the SFJ and the origin of the common trunk for the deep femoral artery was measured as illustrated in Figure 1. When the SFJ was located proximal to the origin of the trunk, the measured distance was expressed as a positive value. When the SFJ was located distal to the origin of the trunk, the measured distance was expressed as a negative value.

\section{Results}

In 29 out of the 47 cases, the deep femoral artery formed a common trunk with the lateral and medial circumflux arteries, so that the type 1 pattern occupied 

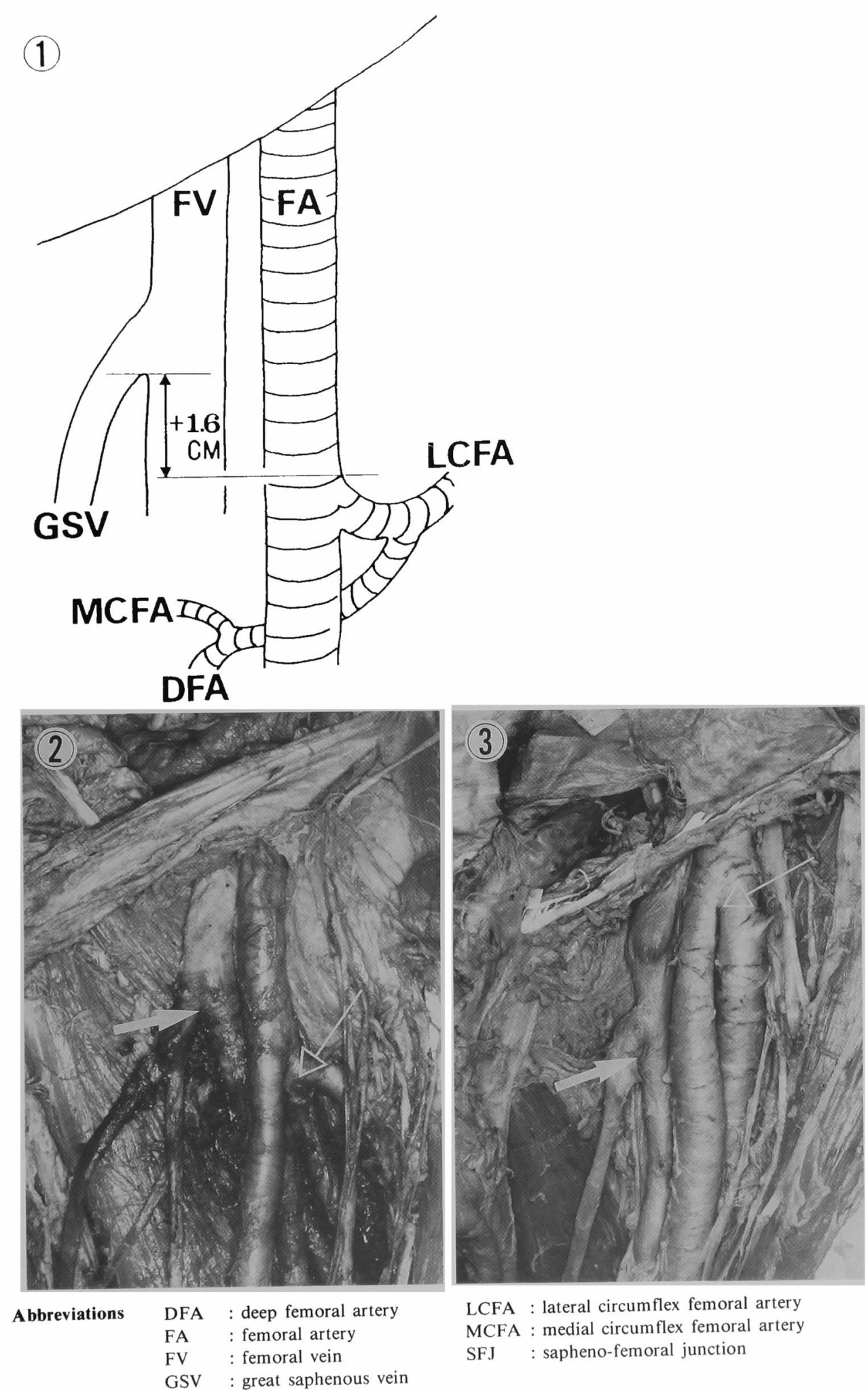

LCFA : lateral circumflex femoral artery MCFA : medial circumflex femoral artery SFJ : sapheno-femoral junction

Fig. 1. Measurement of the distance between the SF J and the common trunk from which the deep femoral artery divides. Note the positive value, when the SFJ is located more proximal to the trunk origin. Fig. 2. The SFJ (arrow) is located proximal to the origin of the common trunk (open arrow). In situ saphenous graft is easy in this case.

Fig. 3. The SFJ (arrow) is located distal to the origin of the common trunk (open arrow). In situ saphenous graft is difficult without stretching the great saphenous vein. 


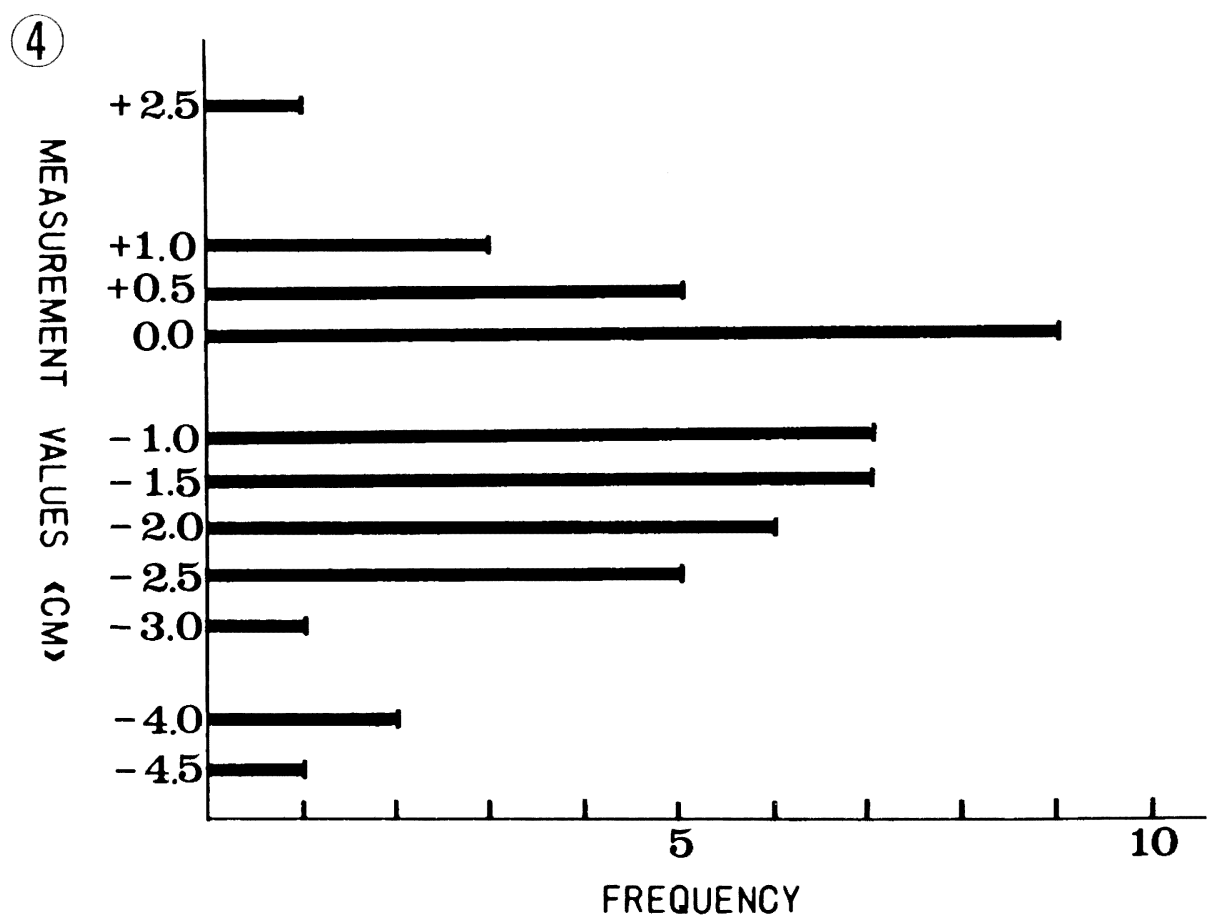

Fig. 4. Distance between the SFJ and the origin of the trunk for the deep femoral artery. Note that there are only 9 positive values.

61.7\%. Among the remaining 18 deep femoral arteries, 12 formed a common trunk with the lateral circumflex femoral artery, while 6 formed a common trunk with the medial circumflex femoral artery. The type 2 pattern thus occupied $38.3 \%$.

As we have found in a previous study, the relative positions of the SFJ's with respect to the origin of the common trunk varied considerably (Figs. 2 and 3). The results of our measurements are summarized in Figure 4. Nine cases $(19 \%)$ had positive values, $9(19 \%)$ were zero, and $29(62 \%)$ had negative values.

\section{Discussion}

In "in situ" grafting the great saphenous vein is cut at the SFJ and at an adequate level distal to an occlusive lesion of the artery. The SFJ is usually anastomosed to the femoral artery proximal to the common trunk from which the deep femoral artery divides, and the other venous end is anastomosed to an intact segment of the artery. There are two reasons why the "in situ" graft sticks at the anastomosis of the SFJ with the femoral artery proximal to the origin of the common trunk. One reason is that the cut end of the SFJ is about equal in diameter to the femoral artery, and this helps to secure a smooth blood flow through the anastomosis. The second reason is that occlusive lesions distal to the trunk origin are often expected to advance up to there, although this does depend on the diseases which cause the arterial occlusion (Haimovici, 1984). According to Gruss (1989), the great saphenous vein is sufficiently long for "In situ" grafting in approximately $80 \%$ of patients. He suggested that the inferior epigastric vein can be utilized to supplement the length in the remaining $20 \%$ of patients. However, our results do not necessarily support this. To anastomose the SFJ to the femoral artery at an appropriate level, the measured value in our study should be positive. In the case of negative values, the anastomosis can not be performed without stretching the great saphenous vein up to the appropriate level, and this could give rise to various postoperative complications such as dehiscence of the anastomosis. Also, use of the inferior epigastric vein may hamper the smoothness of blood flow from the femoral artery to the vein. We obtained only $9(19 \%)$ positive values, suggesting that more than $80 \%$ of patients may not have a sufficient length for "in situ" grafting. It should be pointed out that the effects of fixation and positions of the lower extremity in the cadavers should be taken into account. Although this may be a reasonable analysis, it should not be a reason for excluding more extensive anatomical survey, since the two percentages appear to be very different. 


\section{Acknowledgements}

The authors wish to thank those medical students who practiced dissection during the academic year of 1989 for their cooperation. We are also grateful to Mr. T. Horii, Dr. Y. Fukuo and Dr. S. Morisawa for their helpful suggestions, and to Mrs. M. Shinohara for typing the manuscript.

\section{References}

1) Adachi, B.: Anatomie der Japaner I. Das Arteriensystem der Japaner. Band II. Kaiserlich-Japanischen Universitat zu Kyoto,
Kyoto, 1928.

2) Emura, S., Shoumura, S., Ishizaki, N., Iwasaki, Y., Yamahira, T., Kitamura, Y. and Isono, H.: The anatomical study on the branches of the femoral artery comparison with the findings of Adachi's classification. Acta Anat. Nippon., 60: 623-629, 1985.

3) Emura, S., Shoumura, S., Ishizaki, N., Y amahira, T., Ito, M., $\mathrm{Chen}, \mathrm{H}$. and Isono, $\mathrm{H}$.: The anatomical study on the branches of the femoral artery (II) comparison with the findings of Adachi's classification. Acta Anat. Nippon., 64: 196-205, 1989.

4) Gruss, J. D.: In situ hypass. In: Vascular surgery, pp. 419-431, Springer-Verlag, Berlin, Heidelberg, New York, London, Paris, Tokyo, HongKong, 1989.

5) Haimovici, H.: Femoro-popliteal arteriosclerotic occlusive disease. In: Vascular surgery, 2nd ed., pp. 471-507, 1984. 